\title{
Ionospheric error analysis in GPS measurements
}

\author{
Nicola Crocetto $\left({ }^{1}\right)$, Folco Pingue $\left({ }^{2}\right)$, Salvatore Ponte $\left({ }^{3}\right)$, Giovanni Pugliano $\left({ }^{4}\right)$ and Vincenzo Sepe $\left({ }^{2}\right)$ \\ ${ }^{1}$ ) Dipartimento di Ingegneria Civile, Seconda Università di Napoli, Aversa (CE), Italy \\ $\left(^{2}\right)$ Istituto Nazionale di Geofisica e Vulcanologia, Osservatorio Vesuviano, Napoli, Italy \\ $\left(^{3}\right)$ Dipartimento di Ingegneria Aerospaziale e Meccanica, Seconda Università di Napoli, Aversa (CE), Italy \\ $\left({ }^{4}\right)$ Dipartimento per le Tecnologie, Facoltà di Ingegneria, Università degli Studi «Parthenope», Napoli, Italy
}

\begin{abstract}
The results of an experiment aimed at evaluating the effects of the ionosphere on GPS positioning applications are presented in this paper. Specifically, the study, based upon a differential approach, was conducted utilizing GPS measurements acquired by various receivers located at increasing inter-distances. The experimental research was developed on the basis of two groups of baselines: the first group comprises «short» baselines (less than $10 \mathrm{~km}$ ); the second group is characterized by greater distances (up to $90 \mathrm{~km}$ ). The obtained results were compared either upon the basis of the geometric characteristics, for six different baseline lengths, using 24 hours of data, or upon temporal variations, by examining two periods of varying intensity in ionospheric activity respectively coinciding with the maximum of the 23 solar cycle and in conditions of low ionospheric activity. The analysis revealed variations in terms of inter-distance as well as different performances primarily owing to temporal modifications in the state of the ionosphere.
\end{abstract}

Key words Differential GPS error sources - ionospheric errors - carrier-phase positioning - Geometry-Free Linear Combination - RTK GPS

\section{Introduction}

Atmospheric refraction remains the greatest limitation of GPS in applications requiring centimetric accuracy in real-time. Indeed, the precise positioning via satellite requires special attention to the propagation of GPS signals through the atmosphere yet (Hernández-Pajares et al., 1999), at the same time, the system itself has proven to be a very efficient instrument in

Mailing address: Dott. Giovanni Pugliano, Dipartimento per le Tecnologie, Facoltà di Ingegneria, Università degli Studi «Parthenope», Centro Direzionale, Isola C4, 80143 Napoli, Italy; tel: +39 0815476734; Fax: +39 0815476777; e-mail: giovanni.pugliano@uniparthenope.it observations of the ionosphere (Coster et al., 1992; Lin, 2001).

As is known, by utilzing the GPS RTK differential technique, carrier-phase based positioning results in a rapid and high precision positioning when the ambiguities are fixed to their correct integer values.

As regards real-time differential techniques, the resolution of phase ambiguity is nonetheless strongly influenced by the entity of certain types of GPS measurement errors, especially those involving ionospheric errors. The study highlights a substantial variation in performance identified by the loss of spatial correlation hypothesis validity. In increasing the distances, the OTF resolution procedure for integer phase ambiguity became complicated in terms of time and reliability with repercussions on position accuracy (Wanninger, 1995).

With the aim of obtaining an increment in the resolution capacity of integer phase ambiguity, the primary objective was to reduce the 
differential ionospheric error present in the double difference observable. This error was caused by the fact that certain types of GPS measurement errors, including ionospheric errors, are not adequately reduced by double difference processing, and are thus expressed as differential terms in the double phase equation. With this objective in mind, it was necessary to examine this type of error, understand the behaviour of the cause which might produce it and evaluate the effects on the measurements.

The following are the results of a study of ionospheric error, conducted via the utilzation of GPS measurements collected by continuously operating stations in Campania. It can be observed that the test was conducted on data acquired in two periods of differing ionopheric activity intensity, respectively during the maximum of the solar cycle and in conditions of weak ionospheric activity.

\section{Phenomena of the upper atmosphere}

Modern knowledge of the ionosphere has provided an adequate comprehension of its morphology as well as the basic mechanisms caused by solar radiation. Nonetheless, sensitivity to phenomena of a different nature makes the ionosphere extremely variable. Its very behaviour reveals daily and seasonal variations and depends on the influence of solar activity. The state of the ionosphere also depends on geographic latitude and the geomagnetic field. In addition to the preceding variations, defined as regular variations, there are other irregularities which may occasionally intensify and constitute so-called ionospheric perturbances.

As the very name of the ionosphere indicates, there is a significant concentration of ions and free electrons in the upper strata of the atmosphere. It extends for approximately $50 \mathrm{~km}$ to $1000 \mathrm{~km}$ above the surface of the earth and has a dispersive effect on GPS signal frequencies.

Owing to the effects of refraction, apart from a negligible, curvilinear deviation of the signal trajectory, the ionosphere determines a variation in the speed of propagation. This influence depends on the frequency and is func- tion of the number of electrons along the signal trajectory.

Omitting terms superior to the second order terms, ionospheric error for phase measurements is given in cycles by the following expression (Klobuchar, 1996)

$$
d_{i o n}=-\frac{I}{c f}
$$

where:

I $=40.3$ TEC $\left(\right.$ cycles $\left.\cdot \mathrm{m} / \mathrm{s}^{2}\right)$;

c is the speed of light in a vacuum;

$\mathrm{f}$ is the frequency.

TEC (Total Electron Content) represents the parameter adopted to describe the state of the ionosphere and is the electron content of a $1-\mathrm{m}^{2}$ column along the signal path between satellite $\mathrm{S}$ and receiver $\mathrm{R}$ and is given by the integration of $\mathrm{N}_{\mathrm{e}}$ electron density extended to the entire ionospheric path:

$$
T E C=\int_{R}^{S} N_{e} d s
$$

where:

TEC is expressed in TEC units (TECU) con 1 TECU $=10^{16}$ electrons $/ \mathrm{m}^{2}$;

$\mathrm{Ne}_{\mathrm{e}}$ is the density of electrons (electrons $/ \mathrm{m}^{3}$ ).

Considering that the state of the ionosphere essentially depends upon the sun's electromagnetic radiations, which are responsible for ionization, and corpuscular swarms of solar wind, which produce perturbances, it can consequently be observed that TEC undergoes numerous variations which are caused by solar phenomena (Gao and Liu, 2002; Leitinger et al., 1975; Liao and Gao, 2001).

The pattern of TEC values is characterised by daily, seasonal and eleven-year periodicities which are related to solar activity.

Daily TEC variations are essentially caused by changes in the structure of the ionosphere which occur between night and day. In particular, the trend of electronic density is influenced by solar radiation and by the recombination time. Therefore, as regards daily TEC values, a maximum value is frequently produced one hour after solar noon, generally between 1:00 p.m. and 3:00 p.m. local time; occasionally, an- 
other peak can be recorded during the hours after sunset (Hernández-Pajares et al., 2006).

TEC values also have seasonal variations based upon the months of the year. In the Northern hemisphere, minimum values are recorded during summer and maximum values during both the equinoxes and winter. The entity of TEC may be two to three times greater in winter as opposed to summer.

TEC values also vary according to solar activity and is characterised by an average periodicity of eleven-years. Figure 1 (SIDC, 2006) illustrates the succession of the last five cycles of solar activity.

TEC entity depends upon the cycle phase of solar activity. Cycle 23 began in mid-1996, and maximum activity phases were recorded between 2000-2003. Of specific interest is the typical double peak graph in which, following the maximum in 2000, there was a resumption of activity with a second peak in early 2002 (fig. 1).

In addition to temporal variations, TEC is

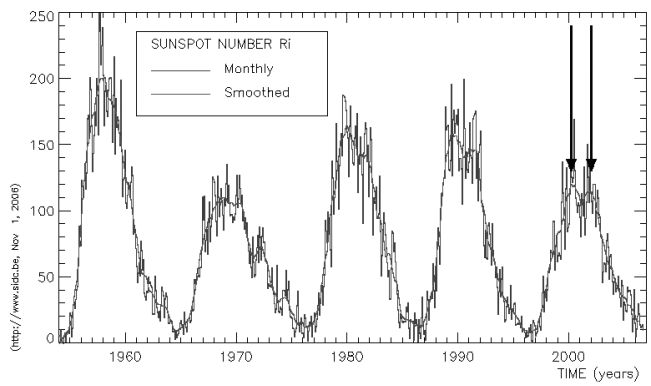

Fig. 1. Succession of cycles of solar activity from 1954 to 2006.

related to geographic and geomagnetic positions. Maximum values are in correspondence to the equatorial anomaly circa $\pm 15^{\circ}$ from the geomagnetic equator (Wielgosz et al., 2004).

TEC variations based upon position are represented by GIM (Global Ionosphere Maps) generated by CODE (Center for Orbit Determination in Europe) which uses data from the IGS

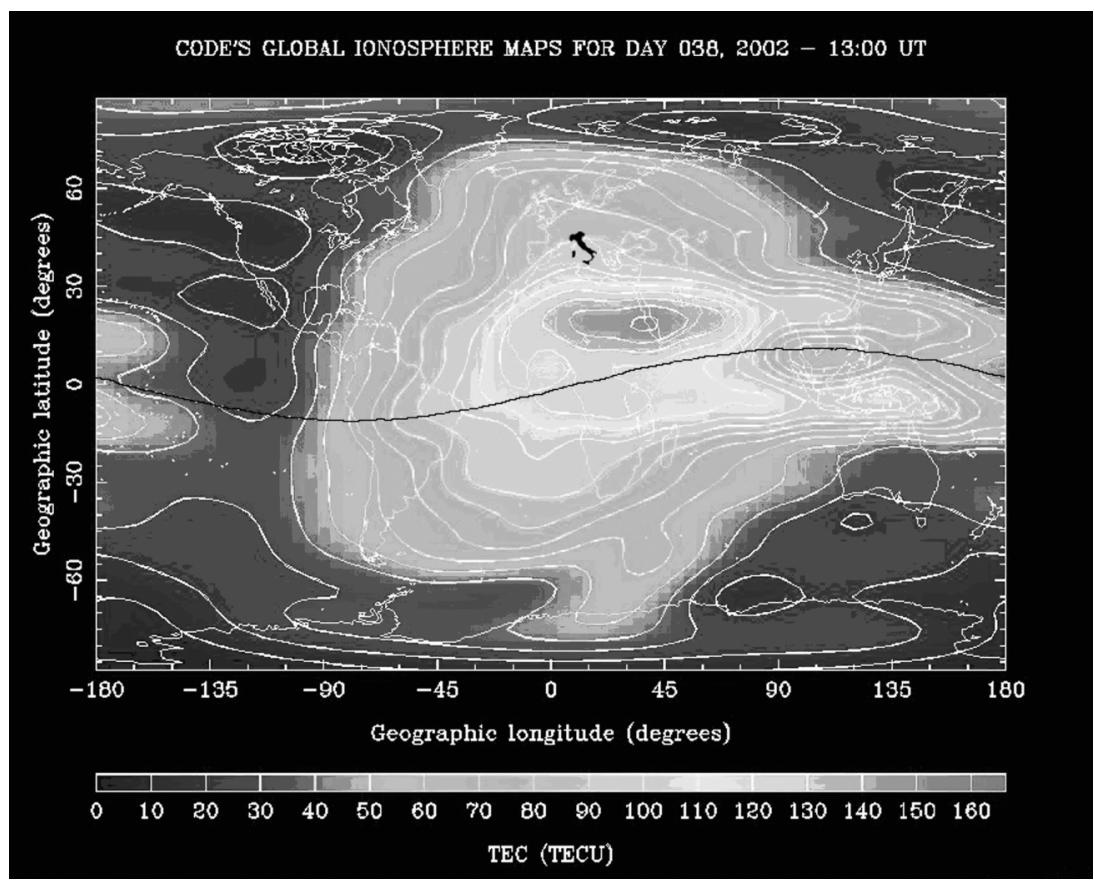

Fig. 2. GIM (Global Ionosphere Map) illustrating VTEC. 
international network of GPS stations (CODE, 2002; Schaer, 1999). Figure 2 illustrates vertical, diurnal TEC values during conditions of intense ionospheric activity. The typical dual «tail» structure travels in an east-west direction along the geomagnetic equator reflecting apparent solar motion around the earth.

There is another factor which interacts with GPS measurements in the ionosphere: the presence of local perturbances within the ionospheric structure causes amplitude fading and phase fluctuation of the received signal. This phenomenon, known as scintillation (Aarons, 1982), has proven to be highly non-correlated in both spatial and temporal terms.

The effects of ionospheric scintillation, especially frequent during periods of maximum solar activity, are predominant in equatorial $\left( \pm 10^{\circ}-20^{\circ}\right.$ geomagnetic latitude), auroral $\left(65^{\circ}\right.$ $\left.75^{\circ}\right)$ and polar $\left(>75^{\circ}\right)$ regions. When effects are also produced at mid-latitudes, it is an extension from the equatorial and auroral regions (Skone, 1998; Skone and De Jong, 1999).

Scintillations are generally recorded during night-time hours, between sunset to mid-night and occasionally even later, in all regions. Furthermore, that there are seasonal variations related to the longitude which produce the greatest effects from September to March in the area extending from the Americas to India, and from April to August in the Pacific region (Wanninger, 1993).

\section{Geometry-Free linear combination for ionospheric delay analysis}

In order to examine ionospheric error, we intend to introduce a mathematical formula capable of expressing the differential delay based upon phase measurements from GPS reference stations.

Among the various combinations of phase measurements derived from the two carrier frequencies L1 and L2 which are prevalently used in GPS models, the geometry-free (Pugliano, 2004) combination has proven to be especially useful in examining ionospheric refraction errors.

By utilzing a double frequency receiver, the
$\Phi_{\mathrm{GF}}$ observable generated by a linear combination of L1 and L2 carrier frequency phase measurements, expressed in meters, can be obtained from the following expression:

$$
\Phi_{G F}=\lambda_{1} \phi_{1}-\lambda_{2} \phi_{2}=\Phi_{1}-\Phi_{2}
$$

where:

$\Phi_{\mathrm{GF}}$ is the geometry-free observable expressed in meters;

$\lambda_{1}, \lambda_{2}$ are the wave lengths of the carrier frequencies (19.03 $\mathrm{cm}$ for L1 and 24.42 cm for L2);

$\phi_{1}, \phi_{2}$ are the phase measurements, respectively, of L1 carrier frequency and the L2 carrier frequency, in cycles;

$\Phi_{1}, \Phi_{2}$ are the phase measurements in meters.

It is observed that geometry-free combination can only be expressed in meters. This peculiarity is in agreement with the fact that the corresponding $\lambda_{\mathrm{GF}}$ wavelength, based upon equation (3.2), is $\infty$.

$$
\lambda\left(\mu_{1}, \mu_{2}\right)=\frac{\lambda_{1} \lambda_{2}}{\mu_{1} \lambda_{2}+\mu_{2} \lambda_{1}}
$$

where:

$\mu_{1}, \mu_{2} \quad$ are the $\mu_{1}=\lambda_{1}$ e $\mu_{2}=-\lambda_{2}$ coefficients.

Equations of phase measurements $\phi_{1}$ and $\phi_{2}$, expressed in cycles, are considered:

$$
\begin{aligned}
\phi_{1}= & \frac{1}{\lambda_{1}}\left(\rho+d \rho+c d t-c d T+d_{\text {trop }}\right)- \\
& -d_{\text {ion } 1}+N_{1}+\varepsilon\left(\varphi_{1}\right) \\
\phi_{2}= & \frac{1}{\lambda_{2}}\left(\rho+d \rho+c d t-c d T+d_{\text {trop }}\right)- \\
& -d_{\text {ion } 2}+N_{2}+\varepsilon\left(\varphi_{2}\right)
\end{aligned}
$$

where:

$\rho \quad$ geometric distance between the satellite and the receiver;

d $\rho$ satellite orbit error;

c speed of light;

dt satellite clock error;

dT receiver clock error;

$\mathrm{d}_{\text {trop }}$ tropospheric error;

$\mathrm{d}_{\text {ion }}$ ionospheric error, in cycles;

$\mathrm{N}$ phase ambiguity (integer number of wavelengths expressed in cycles);

$\varepsilon(\phi)$ phase measurement noise (multipath and receiver noise); 
and considering that $\mathrm{d}_{\text {ion }}$ in meters is given, based upon the equation (2.1), by

$$
d_{\text {ion }}=-\frac{I}{c f} \cdot \lambda=-\frac{I}{c f} \cdot \frac{c}{f}=-\frac{I}{f^{2}}
$$

the geometry-free observable is thus defined, based upon (3.1), by the following expression:

$$
\begin{aligned}
\Phi_{G F}= & \left(\begin{array}{l}
\rho+d \rho+c d t-c d T+ \\
+d_{\text {trop }}-\frac{I}{f_{1}^{2}}+\lambda_{1} N_{1}+\varepsilon\left(\Phi_{1}\right)
\end{array}\right)+ \\
& -\left(\begin{array}{l}
\rho+d \rho+c d t-c d T+d_{\text {trop }}- \\
\frac{I}{f_{2}^{2}}+\lambda_{2} N_{2}+\varepsilon\left(\Phi_{2}\right)
\end{array}\right)= \\
& =-I\left(\frac{f_{2}^{2}-f_{1}^{2}}{f_{1}^{2} f_{2}^{2}}\right)+\lambda_{1} N_{1}- \\
& -\lambda_{2} N_{2}+\varepsilon\left(\Phi_{1}\right)-\varepsilon\left(\Phi_{2}\right)
\end{aligned}
$$

It should be noted that this expression is independent of clocks and geometry (receiver and satellites coordinates), and thus gives origin to the name geometry-free.

The objective is to use this observable for an estimate of ionospheric refraction on phase measurements.

In applying double difference processing, the following is obtained from the equation (3.5)

$$
\begin{aligned}
\Delta \nabla \Phi_{G F}= & -\Delta \nabla I\left(\frac{f_{2}^{2}-f_{1}^{2}}{f_{1}^{2} f_{2}^{2}}\right)+\lambda_{1} \Delta \nabla N_{1}- \\
& -\lambda_{2} \Delta \nabla N_{2}+\Delta \nabla \varepsilon\left(\Phi_{1}\right)-\Delta \nabla \varepsilon\left(\Phi_{2}\right)
\end{aligned}
$$

Considering that for distances greater than $10 \mathrm{~km}$ between receivers, multipath and receiver noise are negligible in comparison with ionospheric error, therefore, the equation (3.6) becomes

$$
-\Delta \nabla I=\frac{f_{1}^{2} f_{2}^{2}}{f_{2}^{2}-f_{1}^{2}}\left(\Delta \nabla \Phi_{G F}-\lambda_{1} \Delta \nabla N_{1}+\lambda_{2} \Delta \nabla N_{2}\right)
$$

Furthermore, from the formula (2.1) the following can be deduced in differential terms

$$
\Delta \nabla d_{i o n_{1}}=-\frac{\Delta \nabla I}{c f_{1}}
$$

Therefore, by substituting equation (3.8) into
(3.7), the following is obtained in cycles

$$
\begin{aligned}
\Delta \nabla d_{i o n_{1}}= & \frac{1}{c f_{1}}\left(\frac{f_{1}^{2} f_{2}^{2}}{f_{2}^{2}-f_{1}^{2}}\right) \\
& \left(\Delta \nabla \Phi_{G F}-\lambda_{1} \Delta \nabla N_{1}+\lambda_{2} \Delta \nabla N_{2}\right)
\end{aligned}
$$

Lastly, the ionospheric differential error for L1 carrier frequency can be obtained, in meters, by multiplying equation (3.9) by the $\lambda_{1}$ wavelength in order to obtain

$$
\Delta \nabla I S_{L 1}=\frac{f_{2}^{2}}{f_{2}^{2}-f_{1}^{2}}\left(\Delta \nabla \Phi_{G F}-\lambda_{1} \Delta \nabla N_{1}+\lambda_{2} \Delta \nabla N_{2}\right)
$$

The definite observable (3.10) is also designated with the term ionospheric signal since residual error, for the most part, can be attributed to the ionosphere.

Given a priori known receiver coordinates and ambiguities between stations, equation (3.10) therefore allows us to express differential ionospheric error through double difference of the geometry-free observable given by

$$
\Delta \nabla \Phi_{G F}=\Delta \nabla \Phi_{1}-\Delta \nabla \Phi_{2}
$$

\section{Analysis of results}

In adopting the above described method, the entity of differential ionospheric error on measurements from seven observation points was evaluated.

An area in the Campania territory was selected as a test zone (fig. 3).

Specifically, six permanent stations as well as a temporary receiver were utilized. Five of the six existing stations belong to the Italian National Institute of Geophysics and Vulcanology - the Vesuvius Observatory and are part of a permanent GPS network in the integrated geodetic monitoring system of the Neapolitan volcanic area (Pingue et al., 2002), the sixth is the permanent station of the «G.C. Gloriosi» Technical Institute in Battipaglia.

The measurements utilzed were taken concurrently from seven different stations at a rate of $30 \mathrm{~s}$, over a 24 hour period.

The experimental research was developed 


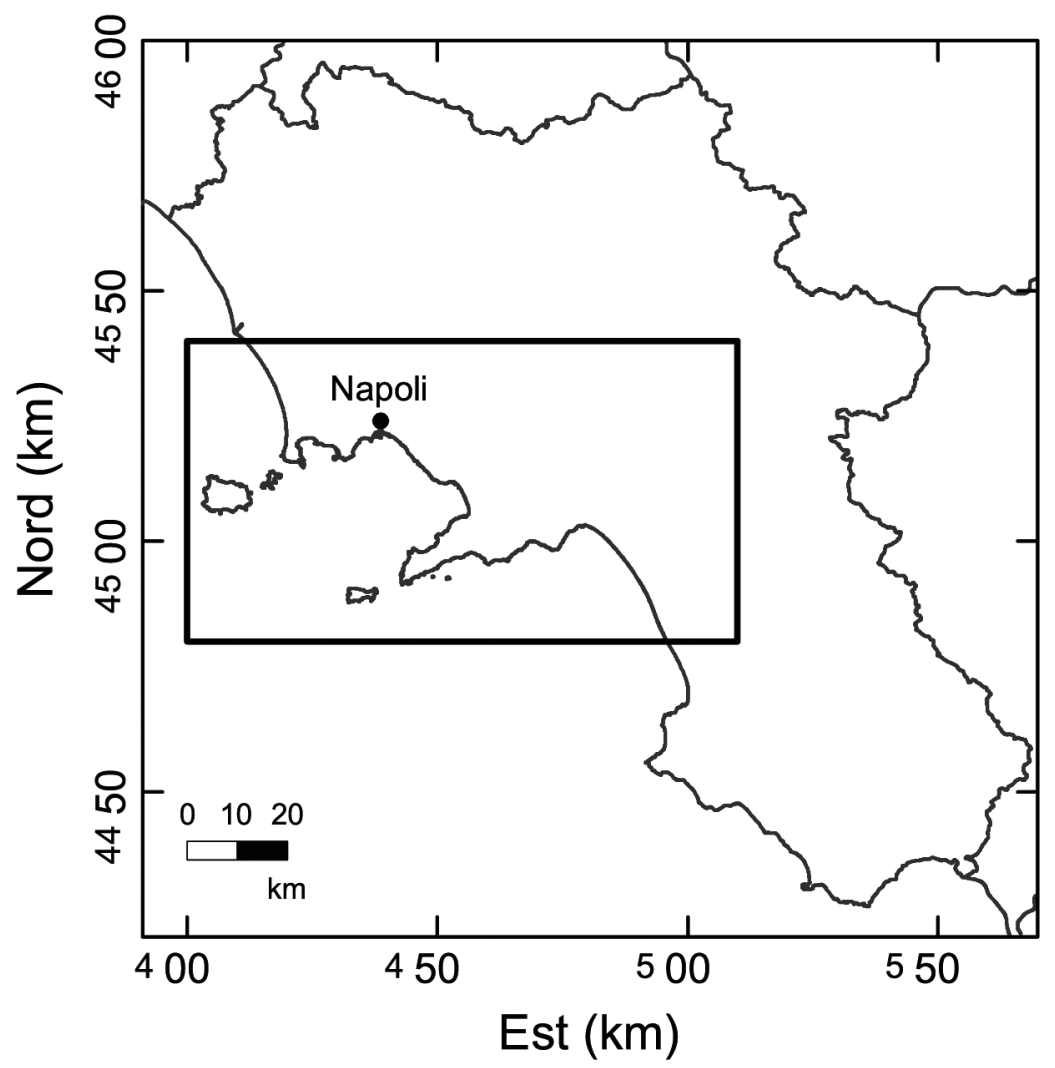

Fig. 3. Test Area.

either upon the basis of the geometric characteristics, on six different baseline lengths, using 24 hours of data, or based upon temporal variations, by examining two days of measurements respectively coinciding with the maximum of the 23 solar cycle on 7 February 2002 and in conditions of low ionospheric activity on 27 April 2006 (fig. 4) (SIDC, 2006).

The baselines selected for comparison with the six different lengths are reported in table I.

Two groups can be observed: the first group is comprised of baselines of less than $10 \mathrm{~km}$ which correspond to the class of «short» baselines (fig. 5).

The second group is characterized by greater distances, the placement of which is illustrated in fig. 6.
Table I. Lengths of the selected baselines.

\begin{tabular}{cc}
\hline \hline Baseline & Length $(\mathrm{km})$ \\
\hline ACAE - RITE & 1.3 \\
BAIA - ACAE & 5.3 \\
BAIA - QUAR & 9.2 \\
QUAR - SERR & 28 \\
BATT - PORT & 60 \\
BATT - SERR & 93 \\
\hline
\end{tabular}

Given the ambiguity between stations as obtained from processing with Bernese soft- 


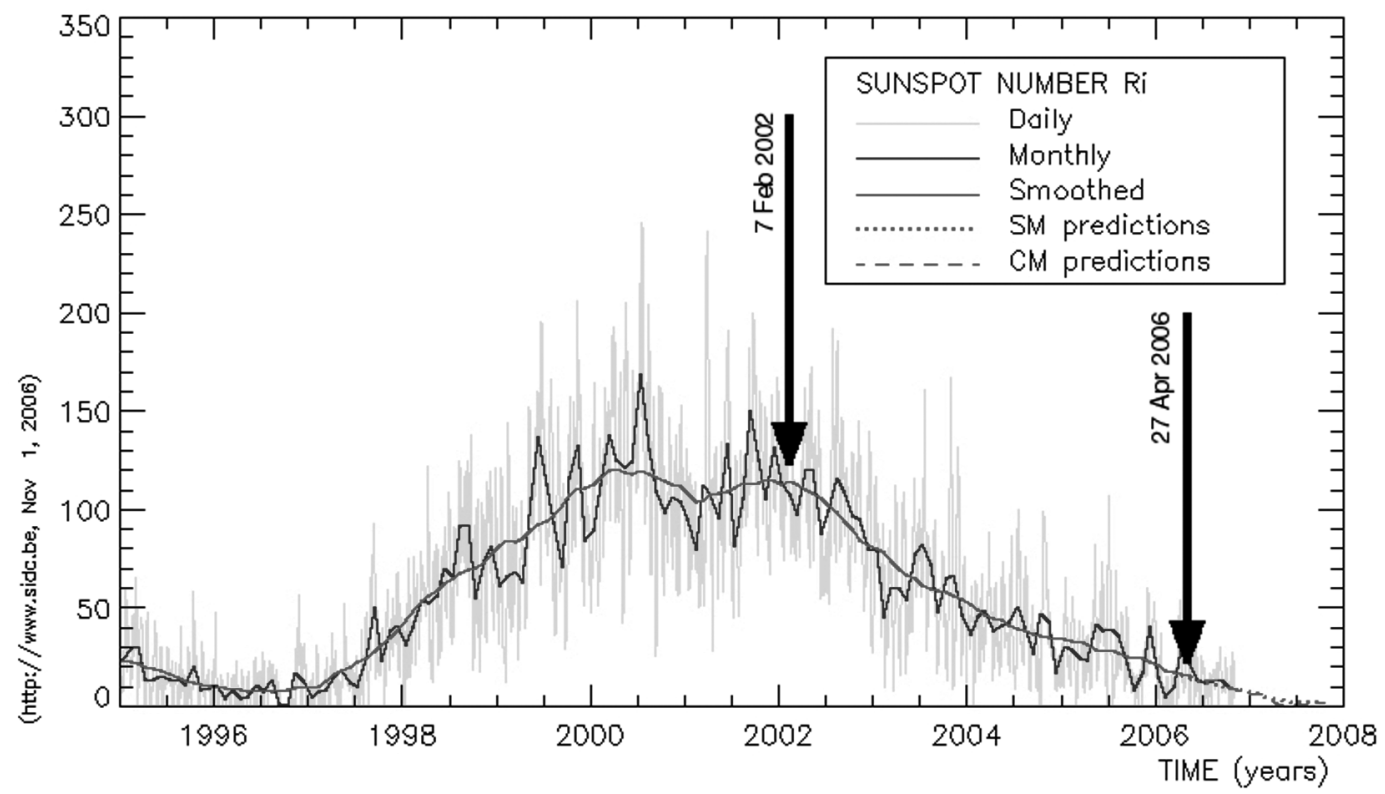

Fig. 4. Solar Activity - Cycle 23.

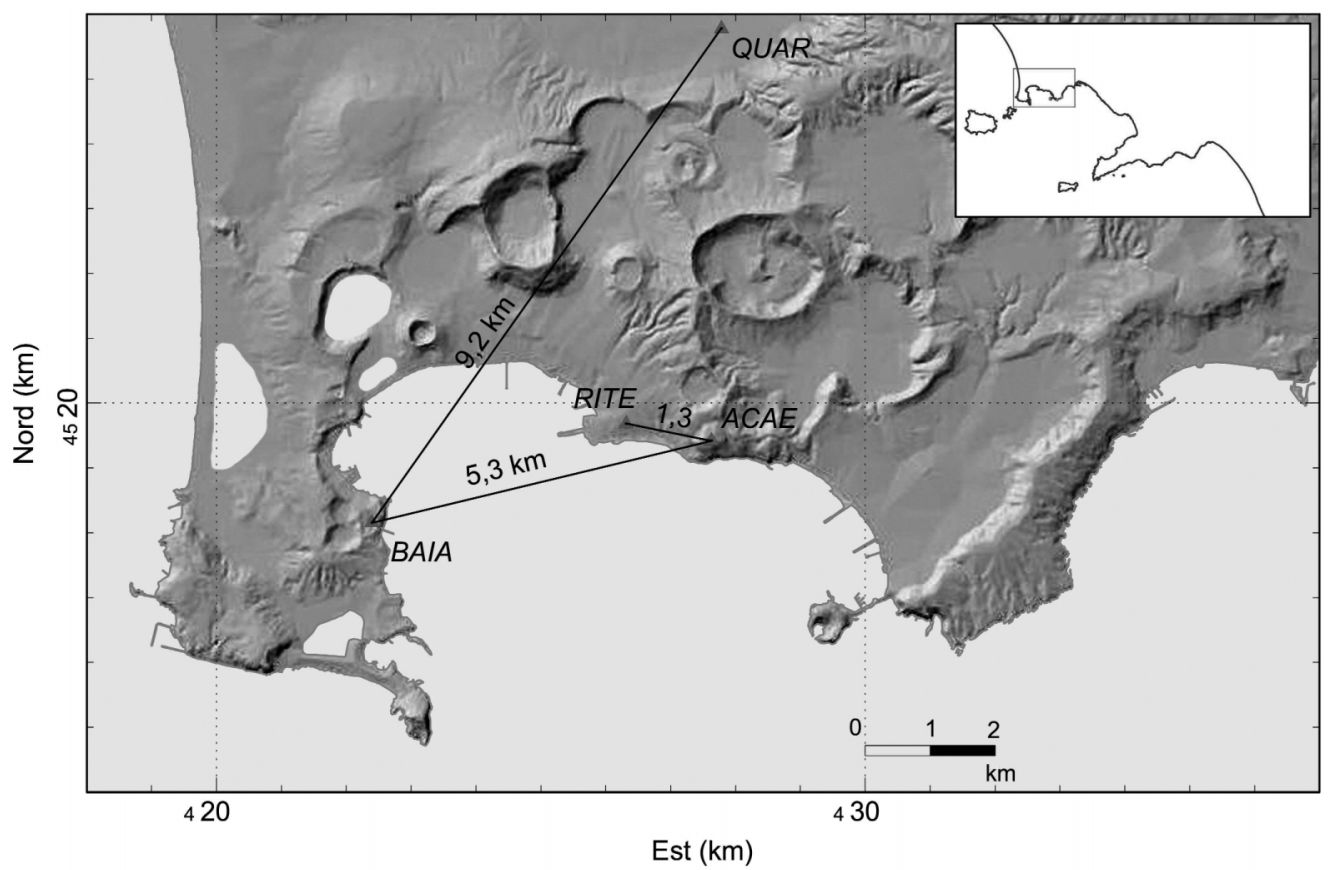

Fig. 5. Location of short baselines. 


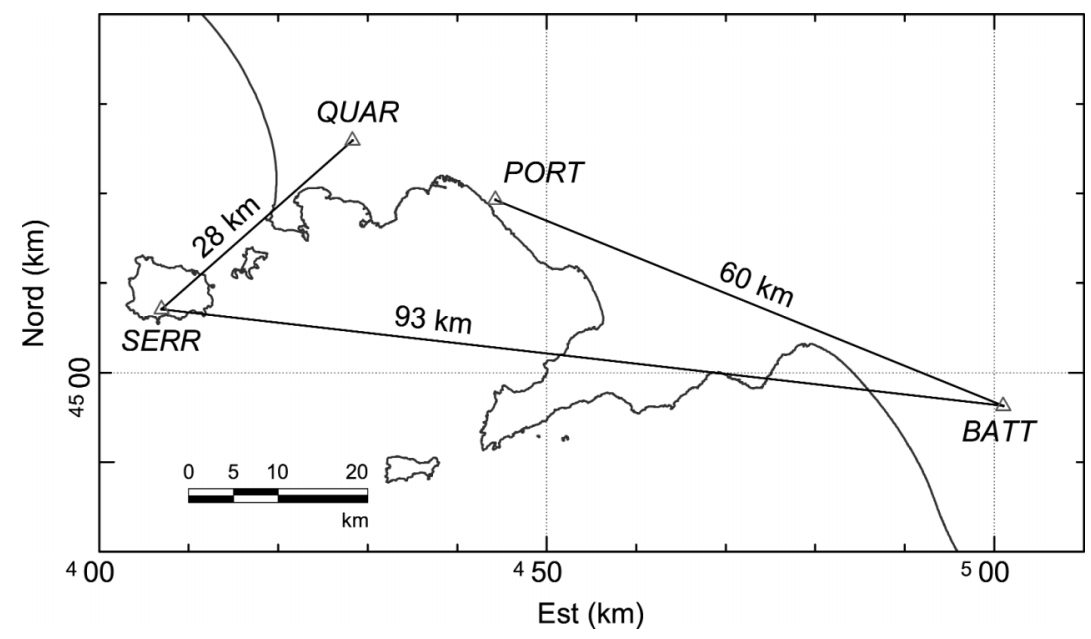

Fig. 6. Location of baselines greater than $10 \mathrm{~km}$.

ware, the equation (3.10) was utilzed.

Figure 7 indicates the differential values of the $\mathrm{IS}_{\mathrm{L} 1}$ observable for the six baselines on 7 February 2002 (black) and on 27 April 2006 (grey).

First of all, in examining the graphics in fig. 7 and comparing the two periods of varying ionospheric activity (black and grey), the correlation between ionospheric activity at different times of the day on 7 February 2002 can be clearly noted, with much higher values occurring during diurnal hours, between 8:00 a.m. and 4:00 p.m. local time. The ionospheric effect is extremely elevated, around $15 \mathrm{ppm}$, in accordance with a particularly active state of the ionosphere for the period under consideration.

Considering, instead, the period of low solar activity (grey), even in distances greater than 10 $\mathrm{km}$, the benefits of a differential approach can

Table II. RMS values and the maximum ionospheric error for the six baselines, respectively, on 7 February 2002 and 27 April 2006.

\begin{tabular}{cccccc}
\hline \hline Baseline & Length $(\mathrm{km})$ & \multicolumn{2}{c}{ RMS (cm) } & \multicolumn{2}{c}{ Max (cm) } \\
& & Feb 2002 & Apr 2006 & Feb 2002 & Apr 2006 \\
\hline ACAE - RITE & 1,3 & 1,5 & 0,8 & 11,2 & 10,2 \\
BAIA - ACAE & 5,3 & 1,4 & 0,8 & 8,3 & 8,5 \\
BAIA - QUAR & 9,2 & 2,0 & 0,8 & 18,9 & 13,6 \\
QUAR - SERR & 28 & 6,3 & 1,4 & 41,5 & 10,0 \\
BATT - PORT & 60 & 12,8 & 3,4 & 86,5 & 15,0 \\
BATT - SERR & 93 & 17,7 & 4,5 & 108,2 & 22,6 \\
\hline
\end{tabular}



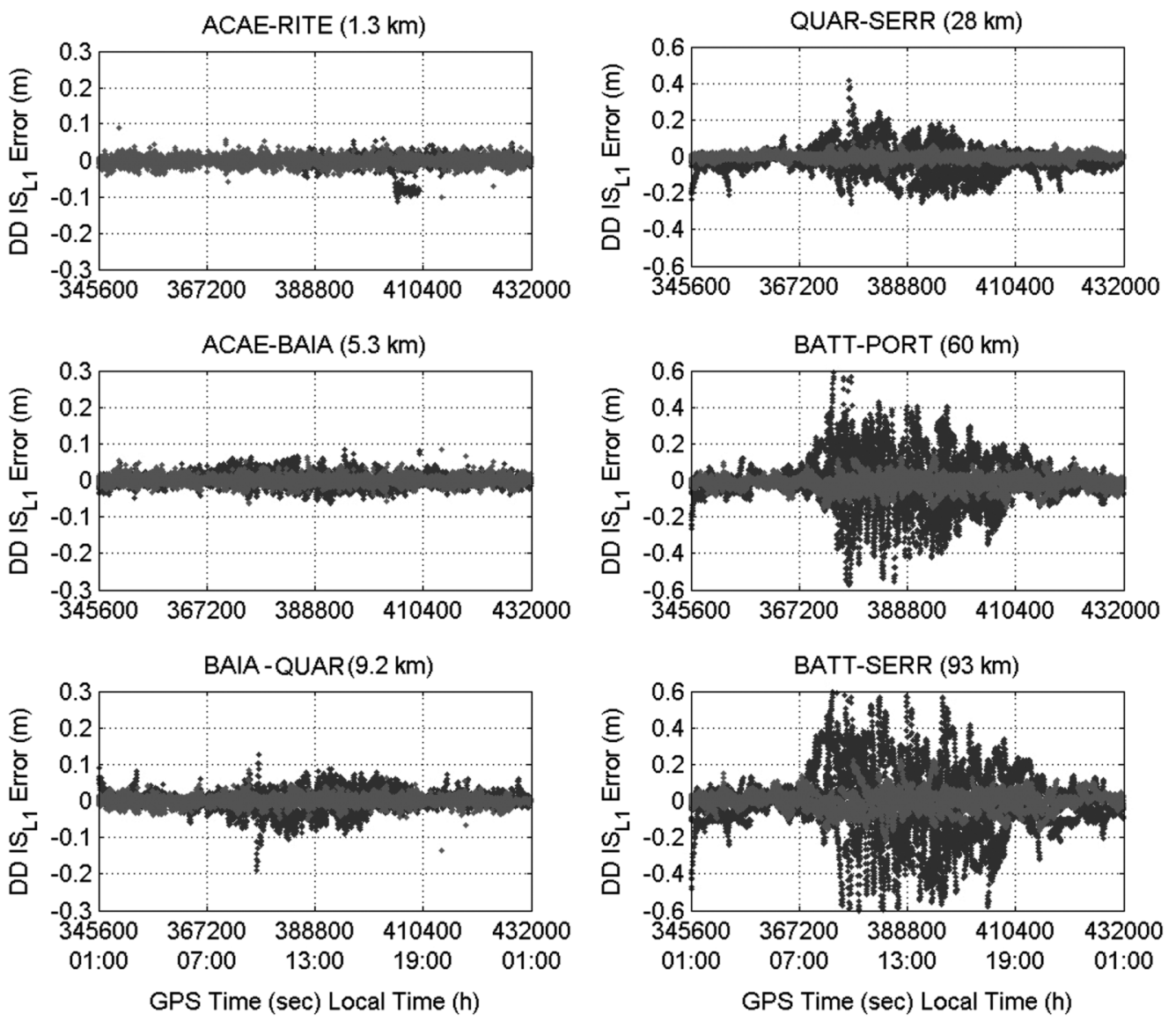

Fig. 7. Ionospheric error, respectively, for 7 February 2002 (black) and 27 April 2006 (grey).

be noted: for the $93 \mathrm{~km}$ baseline, the ionospheric error is reduced in terms of RMS, to $4.5 \mathrm{~cm}$ with a maximum value of $22.6 \mathrm{~cm}$.

The results of comparisons conducted for the measurement days of 7 February 2002 and 27 April 2006 are reported in table II.

Furthermore, these results are represented in fig. 8 in terms of baseline length.

It can be observed that the differing performances are not only associated with variations in distance but primarily reveal appreciable variations owing to temporal modifications in the state of the ionosphere.

The slightly sloping pattern of the graphics corresponding to the period of low solar activity (grey) reveal that, under these conditions, ionospheric behaviour does not vary appreciably from place to place.

During maximum solar activity (black), as distances increase, the trend of the RMS values indicate a reduction in spatial correlation which is maintained only for distances between receivers which are less than $5 \mathrm{~km}$. For the measurements of 2002, the entity of differential ionospheric error grew even less than the $10 \mathrm{~km}$ threshold. However, this behaviour is accentuated when maximum values are taken into consideration. 


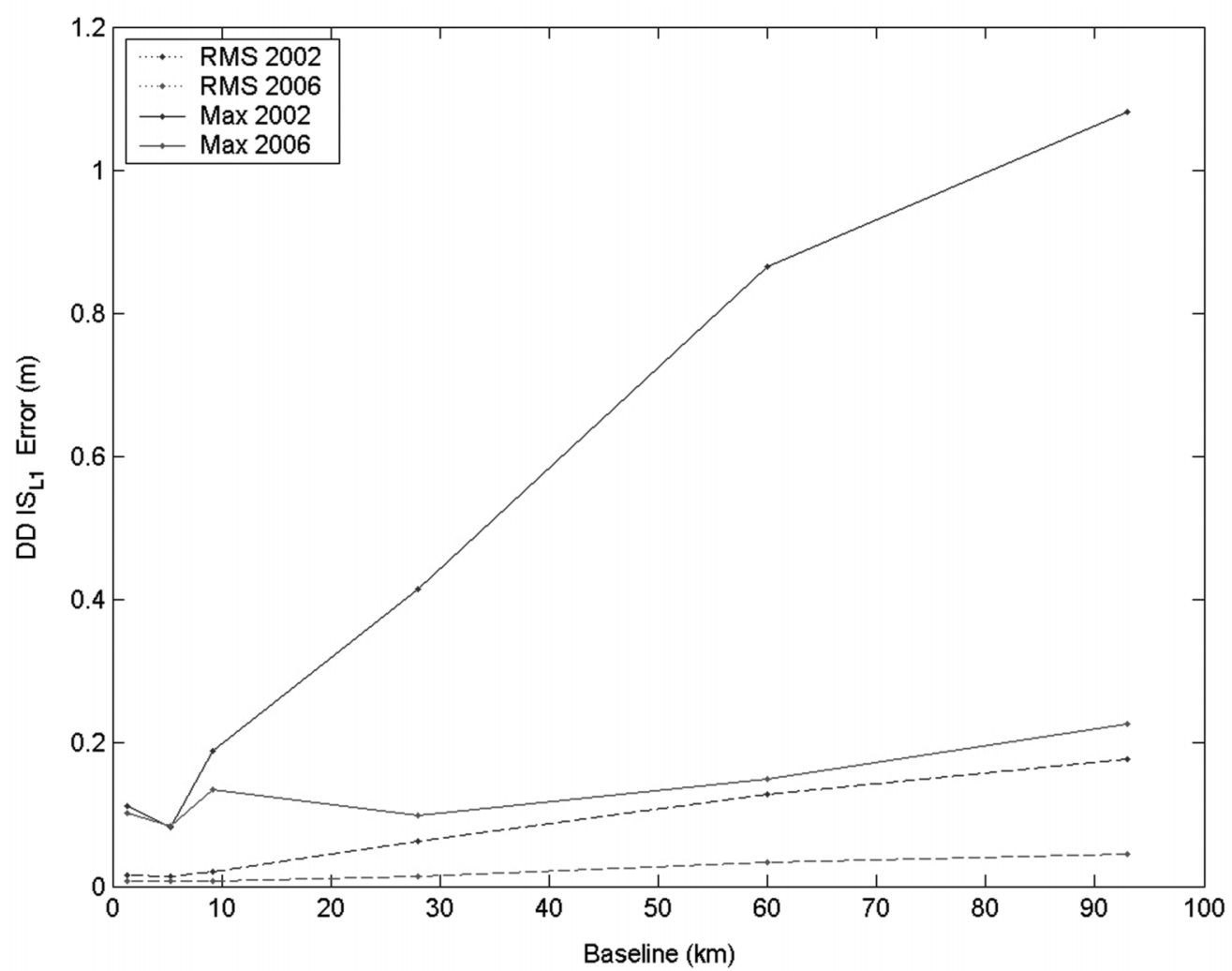

Fig. 8. RMS values and the maximum ionospheric error for the six baselines, respectively on 7 February 2002 and 27 April 2006.

\section{Conclusions}

The processing of data from double frequency GPS receivers allows for monitoring of the ionospheric conditions and, consequently, a far better understanding of the physical phenomena involved.

As regards the most frequently asked question related to the inter-distance between the reference station and the user receiver, it is evident that, owing to the strict relationship between performance and temporal variations in the state of the ionosphere, it is difficult to identify indications of processing time and obtainable precision: the distance between the reference receiver and the user, therefore, represents a highly limiting factor in terms of operativeness.
These drawbacks may be eliminated, or at least reduced, by using of reference station networks which have been viewed with great interest for many years.

Among the aspects to be taken into consideration in approaching these problems, in-depth knowledge of system behaviour in relation to the physical context can not be disregarded: further ionospheric studies are required in order to optimise system use for each, individual case.

\section{REFERENCES}

AARONS, J. (1982): Global Morphology of Ionospheric Scintillations, Proceedings of the IEEE, 70 (4), 360-378.

Code (2002): Global Ionosphere Maps Produced by 
CODE, http://www.aiub.unibe.ch/ionosphere.html, (Global Center for Orbit Determination in Europe, Astronomical Institute, University of Bern).

Coster, A.J., E.M. GaposchKin and L.E. Thornton (1992): Real-time ionospheric monitoring system using GPS, Navigation: Journal of Institute of Navigation, 39 (2), 191-204.

GAO, Y. and Z.Z. LIU (2002): Precise Ionosphere Modeling Using Regional GPS Network Data, Journal of Global Positioning Systems, 1 (1), 18-24.

Hernández-Pajares, M., J.M. Juan, J. Sanz and O.L. Colombo (1999): Precise Ionospheric Determination and its Application to Real-Time GPS Ambiguity Resolution, in Proceedings of Institute of Navigation IONGPS 1999, (Nashville).

Hernández-PAJARES, M., J.M. JuAn and J. SANZ (2006): Medium scale Traveling Ionospheric Disturbances affecting GPS measurements: Spatial and Temporal analysis, Journal of Geophysical Research, 111.

Klobuchar, J. A. (1996): Global Positioning System: Theory and Applications, Vol. I, Cap. 12: Ionospheric Effects on GPS, (American Institute of Aeronautics and Astronautics Inc.), pp. 485-515.

Leitinger, R., G. Schmidt and A. TAuriainen (1975): An evaluation method combining the differential Doppler measurements from two stations that enables the calculation of the electron content of the ionosphere, Journal of Geophysics, 41, 201-213.

LIAO, X. and Y. GAO (2001): High-Precision Ionospheric TEC Recovery Using a Regional-Area GPS Network. Navigation, Journal of Institute of Navigation, 48 (2), 101-111.

LIN, L. (2001): Remote sensing of ionosphere using GPS measurements, in 22nd Asian Conference on Remote Sensing, (Singapore).

Pingue, F., G. Berrino, P. Capuano, C. Del Gaudio, F. Obrizzo, G. P. Ricciardi, C. Ricco, V. Sepe, S. E. P. Borgstrom, G. Cecere, P. De Martino, V. D'Errico, A. La Rocca, S. Malaspina, S. Pinto, A. Russo, C.
Serio, V. Siniscalchi, U. Tammaro and I. Aquino (2002): Sistema integrato di monitoraggio geodetico dell'area vulcanica attiva napoletana: reti permanenti e rilevamenti periodici, Atti della $6^{a}$ Conferenza Nazionale delle Associazioni Scientifiche per le Informazioni Territoriali e Ambientali (ASITA), (Perugia) vol. II, pp. 1751-1764.

Pugliano, G. (2004): Monitoraggio locale della ionosfera attraverso misure GPS, in «Annali della Facoltà di Scienze e Tecnologie», (Università degli Studi di Napoli Parthenope),vol. LXVIII, pp. 87-102.

SchaER, S. (1999): Mapping and Predicting the Earth's Ionosphere Using the Global Positioning System, Ph.D dissertation, (University of Berne).

SIDC (2006): Sunspot Index Graphics, http://sidc.oma.be/index.php3, Sunspot Index Data Center, Royal Observatory of Belgium.

Skone, S. (1998): Wide Area Ionosphere Grid Modeling in the Auroral Region, Ph.D dissertation, (The University of Calgary).

Skone, S. and M. DE Jong (1999): The Impact of Geomagnetic Substorms on GPS Receiver Performance, Proceedings of the International Symposium on GPS, (Tsukuba, Japan).

WANNINGER, L. (1993): Effects of the Equatorial Ionosphere on GPS, GPSWorld, Advanstar Communications, pp. 48-54.

WANNINGER, L. (1995): Improved Ambiguity Resolution by Regional Differential Modeling of the Ionosphere, in Proceedings of Institute of Navigation ION-GPS, (Palm Springs), pp. 55-62.

Wielgosz, P., L.W. Baran, I.I. Shagimuratov and M.V. Aleshnikova (2004): Latitudinal variations of TEC over Europe obtained from GPS observation, Annales Geophysicae, 22, 405-415.

(received February 6, 2008; accepted May 8, 2008) 